\title{
Estudio retrospectivo de 130 pacientes con cáncer prostático organoconfinado tratados con braquiterapia prostática
}

\author{
F. Aguiló Lucía*, J.F. Suárez Novo*, R. Correa Generoso**, A.Mํㅗ Boladeras Inglada**, \\ A. Polo Rubio**, J. Pera Fábregas**, E. Condom Mundo*****, C. Cinos Cope***, F. Guedea Edo**, \\ V. Navarro Pérez****, N. Serrallach Mila*
}

*Servicio de Urología, **Servicio de Oncología Radioterápica, ***Servicio de Física Medica y Protección Radiológica, ****Servicio de Estadística, ****Servicio de Anatomía Patológica. Hospital Universitario de Bellvitge. Instituto Catalán de Oncología. Barcelona.

Actas Urol Esp 2005; 29 (1): 47-54

\section{RESUMEN \\ ESTUDIO RETROSPECTIVO DE 130 PACIENTES CON CANCER PROSTATICO ORGANOCONFINADO TRATADOS CON BRAQUITERAPIA PROSTATICA}

Introducción: La braquiterapia prostática con semillas de $\mathrm{I}^{125}$ está indicada en pacientes con cáncer de próstata organoconfinado. Nuestro objetivo es describir la población tratada en nuestra institución mediante implante permanente con semillas de I'125, las características dosimétricas de la técnica y los resultados preliminares de nuestra serie en cuanto a evolución y toxicidad.

Material y métodos: Entre mayo 2000 y marzo de 2003 fueron tratados 130 pacientes con implante permanente transperineal de semillas de $\mathrm{I}^{125}$. Previamente a todos se les realizó volumetría mediante una ecografía prostática transrectal para determinar la configuración del implante, número de semillas y su localización en la próstata con el fin de obtener una adecuada cobertura del PTV (planed target volume o volumen planificado para tratar). Distribución por estadios: $75,72 \%$ T1c; $24,28 \%$ T2a. Gleason < 6 , 94,24\%. La mediana del PSA pretratamiento 6,38 ng/ml. El volumen prostático mediano fue de $30 \mathrm{cc}$. El 16,67\% de los pacientes cuando los recibimos llevaban tratamiento hormonal previo al implante. La edad mediana fue de 64 años. En cuanto a las características técnicas de los implantes: la mediana de agujas utilizadas fue de 24 (14-35), y la mediana de semillas: 76 (46-111). Finalmente la mediana de la actividad fue de $0,39 \mathrm{mCi} / \mathrm{semilla}$, lo que supuso una actividad total implantada media de $80 \mathrm{mCi}$.

Resultados: Analizamos los 130 pacientes con un seguimiento mínimo de 6 meses. La supervivencia a 1 y 2 años fue del $99,1 \%$ y la supervivencia libre de enfermedad global del $98,9 \%$ al año y del $87,8 \%$ a los dos años La supervivencia libre de enfermedad bioquímica en los pacientes de bajo riesgo eran, al año de $98,8 \%$ y a los dos años del $88,7 \%$. Por el contrario en los de riesgo intermedio eran del $100 \%$ y del $83,3 \%$ respectivamente, aunque el número de pacientes es significativamente menor. Como efectos secundarios agudos relevantes encontramos rectitis leve o GI (escala de la RTOG) en 1,4\% y que necesitó medicación sintomática o GII (escala de la RTOG) en 0,8\%. Se presentó hematuria leve o GI (escala de la RTOG) el 53\%, y que precisaran otras medidas o GII (escala de la RTOG) en el 2,64\%. Finalmente hubo que colocar sonda urinaria por retención aguda el 4,3\%.

Conclusión: La braquiterapia prostática es un procedimiento complejo que exige la participación de un equipo multidisciplinar en su realización. Evita una hospitalización prolongada y permite al paciente recuperar sus actividades cotidianas en un periodo corto de tiempo. Aunque la media de seguimiento es corta, los resultados en cuanto a supervivencia libre de recidiva bioquímica y toxicidad son comparables a los descritos en la literatura. La tolerancia al implante ha sido buena.

Es necesario un mayor seguimiento para poder establecer conclusiones a largo plazo.

Palabras clave: Próstata. Cáncer. Braquiterapia.

\section{ABSTRACT}

RETROSPECTIVE STUDY OF 130 PATIENTS WITH ORGANCONFINED PROSTATE CANCER TRATED WITH BRACHITHERAPY

Introduction: The prostate brachitherapy with I 125 seeds has an indication in patients with organconfined prostate cancer. Our objective is to describe the population trated in our institution with permanent $\mathrm{I}^{125}$ seeds implants, the dosimetric characteristics of the technique and the preliminary results of our group-study in terms of evolution and toxicity.

Material and methods: Between May 2000 and March 2003, a 130 patients with permanent implants of ${ }^{125}$ seeds were trated. Beforehand we did prostate volumetric with transrectum prostate ecography in order to asses the configuration of the implant, number of seeds and their place in the prostate with the objective to get a fine coverage of PTV (planet target volume). Stage distribution: $75.72 \%$ T1c; $24.28 \%$ T2a. Gleason<6, 94\%. The PSA pretreatment average was $6.38 \mathrm{ng} / \mathrm{ml}$. The average prostate volume was 30 cc. The $16.67 \%$ of the patients included had hormonal treatment previously to get the implants. The average age was 64 years. The characteristic techniques of the implants were: the average width of the needle as 24 (14-35) and the average of the seeds 76 (46-111). Finally the average activity was $0.39 \mathrm{mCi} / \mathrm{seed}$, wic means average total implant activity of $80 \mathrm{mCi}$.

Results: We analized 130 patients with average follow up 6 months. A 1 to 2 year surveillance was carried out on $98.9 \%$ and the global free disease surveillance (biochemic relapse) of $98.9 \%$ at the year and of the $87.8 \%$ at the end of the 2 years. The relapse in the low risk patients was, $98.8 \%$ after the first year and $88.7 \%$ at the end of 2 year. On the contrary in the middle risk was of $100 \%$ and $83 \%$ respectively, although the amount of patients in significantly less. As a relevant acute secondary effects we found slight rectitys or GI (RTOG scale) in 1.4 and that needs synthomatic medication or GII (RTOG scale) in $0.8 \%$. We found slide hematuria or GI (RTOG scale) in the $53 \%$ and other measures or GII (RTOG scale) in the $2.64 \%$ was needed. Finally we had to set a urinary prove for acute retention in $4.3 \%$.

Conclusion: The prostate brachiterapy is a complex procedure that needs a multidisciplinary team participation in order to be able to carry out. It aboids a long term hospitalitzation and allows for the patient to have daily activity within a short period of time. Despite the fact of the brief follow-up, the results over biochemical relaps and toxicity were similars to the ones in the literature. Tolerance to the implant was good. It would necessary a longer follow-up in order to be able to come to long term conclusions. Key words: Prostate. Cancer. Brachitherapy. 
$\mathrm{E}$ n la actualidad uno de los mayores problemas que tiene planteada la sociedad desde el punto de vista oncológico es la detección precoz del cáncer ${ }^{1,2}$. En el varón el incremento de diagnósticos de cáncer de próstata en estadios iniciales ha cambiado radicalmente desde que se instauró la detección del antígeno prostático específico (PSA) como un parámetro más en las detecciones analíticas habituales a la población masculina a partir de los 50 años ${ }^{3}$.

Los datos de la Asociación Española de Urología estiman que un millón y medio de varones, con edades comprendidas entre los 50 y 75 años, corren el riesgo de padecer cáncer de próstata, y según la International Agency for Research on Cancer, España presenta una tasa ajustada a la población estándar mundial de 20.22 nuevos casos / 100.000 hombres / año ${ }^{4,5}$. Usando la fórmula de la prevalencia [prevalencia $=$ incidencia $\mathrm{x}$ duración de la enfermedad] (duración media entre 5-15 años), la prevalencia en nuestro país sería de 101-300 / 100.000 habitantes. En estudios de autopsias, la prevalencia es más alta con proporciones del $38 \%$ en personas entre 50-70 años, del $41 \%$ entre $70-80$ años y del $57 \%$ en mayores de 80 años. A través de estos datos se confirma la experiencia clínica de que hay muchos varones que mueren con cáncer de próstata pero no de cáncer de próstata ${ }^{6}$.

Pese a que la historia natural del cáncer de próstata no es bien conocida, es evidente que la progresión del proceso en la mayoría de los casos se produce de forma lenta, por lo que muchas lesiones cancerosas pueden permanecer latentes y sin diagnosticar durante muchos años. Sin embargo, una minoría de cánceres prostáticos progresan con rapidez, invaden los tejidos vecinos y metastatizan, principalmente en los huesos .

Los datos de la American Cancer Society indican que el 89\% de los hombres con el diagnóstico de cáncer de próstata sobreviven al menos 10 años. Así, el 58\% de todos los cánceres de próstata se diagnostican cuando todavía están confinados a la próstata, y su índice de supervivencia relativa a los 5 años es del 100\% hayan recibido tratamiento o no. Por otro lado, el 31\% de los cánceres de próstata ya se han propagado localmente con invasión periprostática en el momento del diagnóstico y el índice de supervi- vencia a los 5 años es del 94\%. Finalmente, el $11 \%$ de los cánceres de próstata se diagnostican con metástasis y tienen un índice de supervivencia a los 5 años del 31\%.

La mayoría de los casos de cáncer de próstata en las primeras fases no tienen sintomas clínicos y se detectan por las elevaciones del antígeno prostático específico (PSA) en sangre o por la palpación casual de un nódulo duro prostático en el curso de un tacto rectal rutinario.

La braquiterapia prostática con semillas de I $^{125}$ esta indicada en pacientes con cáncer de próstata en estadios iniciales (Tabla 1). Las tablas de Partin ${ }^{7}$ y los resultados clínicos sugieren que aquellos pacientes con tumores T1-T2a, PSA $<10$ y Gleason $<6$ son buenos candidatos para implante exclusivo de braquiterapia prostática ${ }^{7-9}$.

El $\mathrm{I}^{125}$ es el isótopo radiactivo más usado en braquiterapia prostática. Emite fotones de baja energía $(27 \mathrm{KeV})$, tiene una vida media de 60 días y una baja tasa de dosis entre 7-10 centiGreys/ hora $^{8}$.

Esta serie representa la experiencia de nuestro centro con los actuales métodos de implante. Los resultados son analizados en función de los resultados de PSA y comparados con series clásicas.

\section{MATERIAL Y METODOS}

Nuestro objetivo es describir la población tratada en nuestra institución mediante implante permanente con semillas de $\mathrm{I}^{125}$, valorando las

Tabla 1

Los criterios de selección para braquiterapia exclusiva son:

- Estadio clínico T1-T2a

- Gleason menor de 6

- PSA menor de $10 \mathrm{ng} / \mathrm{ml}$

\section{Contraindicaciones relativas:}

Por presentar mayor riesgo de complicaciones:

- Lóbulo medio agrandado

- Irradiación pélvica previa

- Presencia de síntomas obstructivos bajos

- Riesgo quirúrgico elevado

Por presentar dificultades técnicas:

- Resección transuretral previa

- Tamaño prostático por encima de 50 cc.

- Lóbulo medio prominente

\section{Contraindicaciones absolutas:}

- Expectativa de vida inferior a 5 años

- Riesgo quirúrgico inaceptable

- Metástasis a distancia

- Próstatas voluminosas 
características dosimétricas de la técnica y comentando los resultados preliminares de nuestra serie en cuanto a evolución y toxicidad.

Entre julio de 2000 y marzo de 2003 se han tratado en nuestro centro 343 pacientes afectos de carcinoma de próstata órganoconfinado. De éstos se han realizado 203 prostatectomías radicales y 140 braquiterapias ya que fueron considerados de bajo o intermedio riesgo. Ninguno de éstos había recibido radioterapia externa previa.

Todos los pacientes fueron valorados por un comité multidisciplinar formado por urólogos, oncólogos radioterapeutas, oncólogos médicos y patólogos involucrados en cáncer de próstata. A todos se les realizó un estudio consistente en exploración física con tacto rectal, analítica completa, PSA y ecografia transrectal. Las muestras de biopsia realizadas fuera de nuestro centro fueron revisadas por el mismo uropatólogo.

Previo al implante los pacientes fueron sometidos a un estudio ecográfico prostático transrectal para determinar exactamente la volumetría prostática. Se realizó en posición de litotomía forzada. Se realiza bajo sedación, utilizando el modelo Cheetah 2003 de B\&K.

El transductor se coloca sobre un sistema de posicionamiento calibrado (AccuSeed System ${ }^{\circledR}$ ), de forma que quede fijo. Este soporte permite realizar secciones ecográficas cada $5 \mathrm{~mm}$, desde la base hasta el ápex. En cada una de las secciones se delimita el perímetro de la próstata. El ecógrafo lleva un software incorporado que permite el cálculo del volumen. Posteriormente estas imágenes son transferidas a un sistema de planificación informatizado (Plato-Nucletron).

Seguidamente se realiza un TAC pélvico para determinar la angulación de las ramas isquiopubianas y ver la interferencia que pueda tener el arco púbico sobre la próstata, lo que decide si es posible o no realizar el implante.

La dosimetría clínica se realiza siguiendo las recomendaciones del Task Group 64 y 43 de la American Asoaciation of Physicist in Medicine. Siempre que sea posible se prefiere un diseño de carga periférica con una separación mínima de 1 $\mathrm{cm}$ para intentar cubrir con la isodosis de $145 \mathrm{~Gy}$ la glándula y $0,5 \mathrm{~cm}$ de margen, teniendo en cuenta no superar las dosis de tolerancia de recto y uretra $^{10-13}$.
El implante se realiza unas tres semanas después de la volumetría. En este momento es absolutamente necesario reproducir exactamente las mismas secciones prostáticas obtenidas el día de la volumetría, por lo que hay que reproducir la posición de la próstata. Una vez localizada la próstata con el transductor intrarectal y en la misma posición que la volumetría se coloca la rejilla multiperforada sobre el periné y se van ubicando las semillas en las coordenadas preestablecidas. El implante es controlado en todo momento mediante ecografía transrectal y radioscopia. Una vez finalizado se realiza una cistoscopia para verificar que no hay semillas en vejiga.

El paciente permanece ingresado hasta el día siguiente para vigilar la aparición de complicaciones agudas. La sonda urinaria se retira en 24 horas y se comprueba la correcta diuresis.

Una vez dado de alta todos los pacientes siguen controles periódicos en la Unidad Funcional de Próstata, cada tres meses durante el primer año y semestralmente a partir de entonces, mediante exploración física y PSA. Consideramos recidiva tres elevaciones sucesivas de PSA después de haber conseguido el nadir postimplante, según definición de la ASTRO ${ }^{7,14}$.

\section{RESULTADOS}

De los 140 pacientes tratados solamente analizamos 130 pacientes que son los que llevan una evolución mínima de 6 meses. La mediana de edad es de 64 años. La distribución por estadios se indica en la Tabla 2. Respecto al PSA el 93,6\% tenía un valor de PSA menor de $10 \mathrm{ng} / \mathrm{ml}$, y la media de PSA pretratamiento fue de $6,38 \mathrm{ng} / \mathrm{ml}$.

Tabla 2

\begin{tabular}{cc}
\hline Estadios clinicos: & \\
T2a & $24,28 \%$ \\
T1c & $75,72 \%$ \\
Gleason Score: & \\
GS 2 & $0,67 \%$ \\
GS 3 & $4,03 \%$ \\
GS 4 & $10,47 \%$ \\
GS 5 & $31,54 \%$ \\
GS 6 & $51,01 \%$ \\
GS 7 & $2,01 \%$ \\
PSA : & \\
× $10 \mathrm{ng} / \mathrm{ml}$ & $93,29 \%$ \\
$>10 \mathrm{ng} / \mathrm{ml}$ & $6,71 \%$ \\
\hline
\end{tabular}


La distribución respecto al Gleason fue GS 2: 0,64\%; GS 3: 3,9\%; GS 4: 10,3\% ; GS 5: $30,2 \%$; GS 6: 49,4\%; GS 7: $2 \%$ y no se dispuso de este dato en el 3,9\%. Establecimos dos grupos de riesgo de recidiva bioquímica: Bajo riesgo, aquellos con PSA $<10$ y Gleason $<6(90,7 \%)$ y riesgo intermedio, aquellos con Gleason 7 y PSA entre $10-20(9,23 \%)$.

El $82 \%$ de los pacientes tenían un volumen prostático menor de $50 \mathrm{cc}$. El 16,7\% de los pacientes acudieron a nuestro centro con tratamiento hormonal prescrito previamente a la valoración. $\mathrm{Hu}-$ bo un $8,46 \%$ de pacientes que no se consideraron candidatos al implante por volumen prostático aumentado, y que después de informar de las opciones terapéuticas disponibles optaron por realizar hormonoterapia reductora previa a nueva volumetría ya que querían realizarse una Braquiterapia.

Se emplearon una mediana de 24 agujas por implante (14-35) para colocar una mediana de 76 (46111) semillas. La actividad por semilla fue de 0,391 mCi y la actividad total implantada 30,9 mCi (2043,4). La dosis administrada fue de $145 \mathrm{~Gy}$. La mediana de seguimiento ha sido de 13 meses (6-37).

Se han dividido los pacientes en función del riesgo de recidiva bioquímica encontrando que el $90,7 \%$ eran de bajo riesgo y el 9,3\% de riesgo intermedio. No se ha tratado ningún paciente de alto riesgo.

La supervivencia a 1 y 2 años fue del 99,1\% (Fig. 1) y la supervivencia libre de enfermedad global del $98,9 \%$ al año y del $87,8 \%$ a los dos años (Fig. 2). La supervivencia libre de enfermedad bioquímica en los pacientes de bajo riesgo eran del $98,8 \%$ al año y $88,7 \%$ a los dos años respectivamente (Fig. 3), mientras que los de riesgo intermedio eran del $100 \%$ y del $83,3 \%$ respectivamente (Fig. 4), aunque este dato es poco valorable por el escaso número de pacientes.

La morbilidad después de esta serie de implantes fue baja. Un 4,35\% de pacientes realizaron retención aguda de orina que requirió sonda urinaria, aunque fue retirada en una mediana de 10 días. El efecto secundario más común fue la hematuria postimplante, que fue grado I (RTOG) o leve en el 53,04\% y grado II (RTOG) o que necesitaran otras medidas en el 2,64\%. La frecuencia de rectitis gradol leve fue de $1,4 \%$ y grado II o que precisara medicación en el 0,8\%.

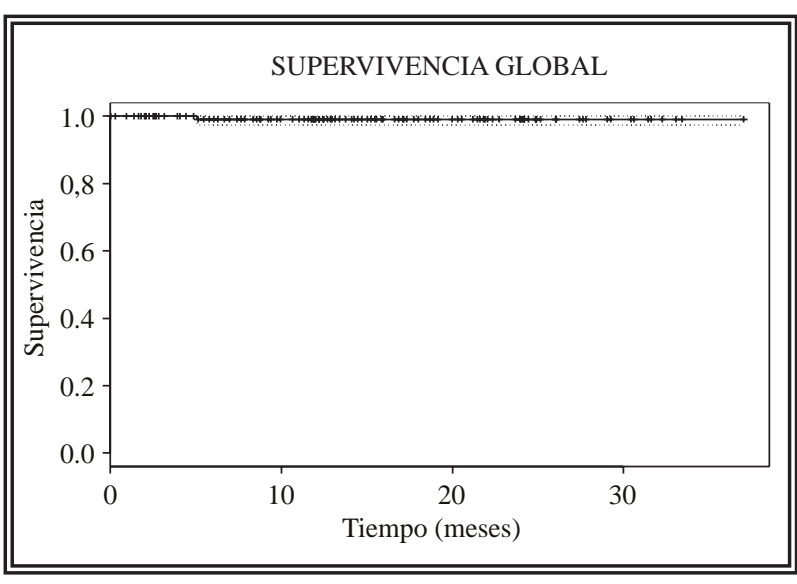

FIGURA I.

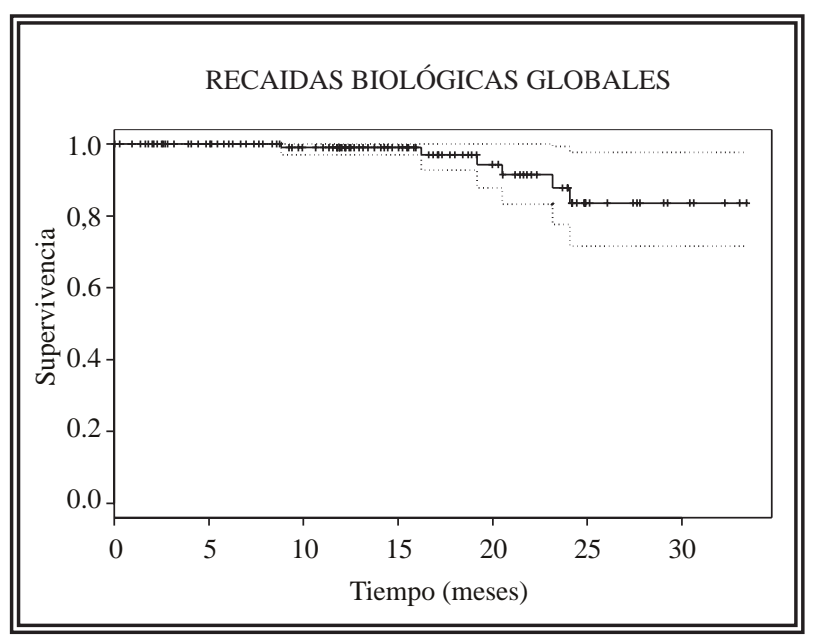

FIGURA 2.

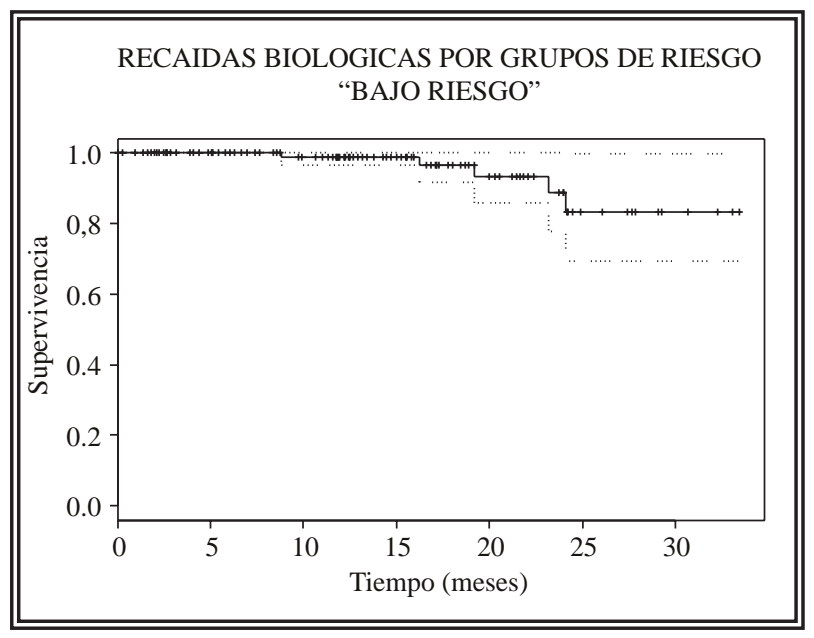

FIGURA 3.

\section{DISCUSIŌN}

La elección del tratamiento óptimo para el cáncer de próstata órganoconfinado es controvertido ya que no existen estudios prospectivos 


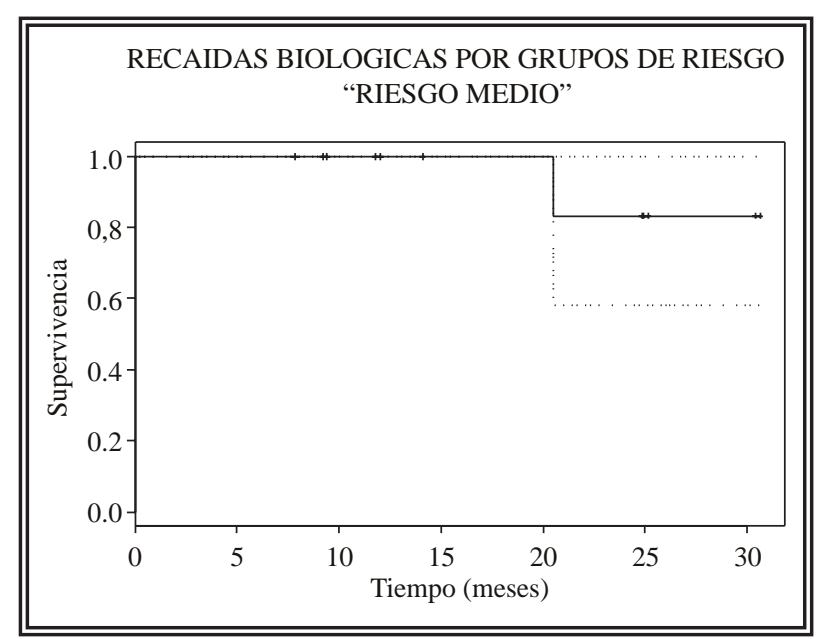

FIGURA 4.

randomizados que comparen distintas alternativas terapéuticas para grupos similares de pacientes. La braquiterapia prostática es un procedimiento que comenzó a utilizarse a principios del siglo pasado y que ha conocido un renacimiento a partir de 1980 gracias a la descripción de la técnica de implante transperineal bajo guía ecográfica transrectal y su posterior optimización ${ }^{1,3}$, ya que permite aumentar la conformidad y la homogeneidad de la distribución de dosis. Por otro lado hay que tener muy en cuenta los prometedores resultados clínicos provenientes del grupo de Seattle ${ }^{1-3}$.

La braquiterapia como monoterapia para el cáncer de próstata órganoconfinado se puede realizar mediante implante permanente, con semillas de $\mathrm{I}^{125}$ o $\mathrm{Pd}^{103}$, o mediante implante temporal con alta tasa de dosis (HDR) utilizando $\operatorname{Ir}^{192}$.

En un trabajo de Wallner et al. ${ }^{25}$ se analiza de forma prospectiva y randomizada la supervivencia libre de enfermedad de pacientes afectos de adenocarcinoma de próstata de bajo riesgo tratados con implante permanente de $\mathrm{I}^{125}$ versus $\mathrm{Pd}^{103}$. Se considera recidiva un $\mathrm{PSA} \geq 0,5 \mathrm{ng} / \mathrm{ml}$ o un nadir de PSA > 0,5 ng/ml. Si bien el periodo de seguimiento es corto, el análisis se hace a los 3 años, no existen diferencias estadísticamente significativas entre el grupo tratado con uno $u$ otro isótopo.

Existe una limitada experiencia publicada en cuanto al tratamiento con HDR monoterapia del cáncer de próstata órgano-confinado.
Los trabajos publicados abundan más en los efectos secundarios y la calidad de vida que en los resultados de supervivencia libre de enfermedad. Yoshioka et al. ${ }^{26}$ analizan los resultados de una serie de 43 pacientes tratados con HDR monoterapia entre 1995 y 2001.

El grupo es altamente heterogéneo, incluyendo pacientes de bajo, intermedio y alto riesgo, con y sin hormonoterapia previa. El seguimiento medio es de 24 meses (rango 1-76), siendo la supervivencia libre de enfermedad a los 3 años del 94\%, 100\% y 55\% respectivamente. Esta heterogenicidad del grupo hace difícil obtener conclusión alguna en lo que respecta a la supervivencia cáncer específica y supervivencia libre de enfermedad, centrándose los autores en el análisis de la toxicidad, tanto aguda como crónica.

Toxicidad rectal aguda grado 4,2 y 1 de la RTOG fue presentada en un 2\%, 28\% y 19\% respectivamente, mientras que 5 de los 43 pacientes presentaron toxicidad tardía, uno grado 2 y el resto grado 1 .

Más recientemente, Martínez et $\mathrm{al}^{27}$ publican un estudio prospectivo en el que comparan la supervivencia libre de enfermedad y la toxicidad aguda y crónica de dos cohortes de pacientes, una tratada con $\mathrm{Pd}^{103}$ (84 pacientes) y otra tratada con HDR (65 pacientes). Se trataba de pacientes de bajo e intermedio riesgo, habiéndose utilizado hormonoterapia para disminuir el tamaño de la glándula en un 36\%.

El seguimiento medio es de 35 meses, no existiendo diferencias estadísticamente significativas entre ambos grupos en cuanto a estadio clínico, PSA, Gleason, utilización de hormonoterapia, volumen prostático, sintomatología miccional previa ni tiempo de seguimiento. La supervivencia libre de enfermedad bioquímica a los 3 años fue del 97\% para el grupo de $\mathrm{Pd}^{103}$ y del 98\% para el grupo de HDR, según criterios ASTRO.

En cuanto a toxicidad aguda fue significativamente menor el en grupo de HDR en cuanto a disuria, frecuencia/urgencia y dolor rectal, no existiendo diferencia alguna en el porcentaje de hematuria, retención aguda de orina, rectorragias o diarrea. En cuanto a la toxicidad crónica únicamente existe diferencia entre ambos grupos en lo que concierne a la frecuencia/urgencia, que 
es menor en el grupo de HDR. El porcentaje actuarial de disfunción eréctil a los 3 años en pacientes previamente potentes y a los que no se somete a hormonoterapia previa es del 35\% para la serie global, siendo significativamente inferior en el grupo de HDR ( $45 \%$ versus $16 \%, p=0,062$ ).

Recientemente numerosas instituciones han publicado los resultados de sus series de braquiterapia con implante permanente de semillas. La comparación entre dichas series es difícil en muchos casos debido a la variabilidad en los criterios de inclusión de los pacientes, la utilización de hormonoterapia o radioterapia neoadyuvante y las diferencias en la definición de la recidiva bioquímica. Zelefsky et al. en una serie de 248 pacientes tratados entre 1989 y 1996 reporta una supervivencia libre de recidiva bioquímica a cinco años para pacientes de bajo riesgo (estadio T2a o menor y GS $<7$ y PSA inicial $<10 \mathrm{ng} / \mathrm{ml}$ ), riesgo intermedio (un incremento en el valor de alguno de estos indicadores) y alto riesgo (un incremento en el valor de dos o más de estos indicadores) de $88 \%, 77 \%$ y $38 \%$ respectivamente ${ }^{9}$. Stock et al. reportan una serie de 243 pacientes tratados entre 1990 y 1996.

La estratificación de grupos de riesgo es como sigue: grupo de bajo riesgo (estadio T2a o menor, GS $<7$ y PSA inicial $<10 \mathrm{ng} / \mathrm{ml}$ ), grupo de riesgo intermedio (estadio T2b, GS $=7$ o PSA inicial entre 10,1-20 ng/ml) y grupo de alto riesgo (dos o mas factores de riesgo intermedio o GS $>7$ o PSA inicial > $20 \mathrm{ng} / \mathrm{ml}$ ).

La supervivencia libre de recidiva bioquímica a ocho años es de $88 \%, 81 \%$ y $65 \%$ respectivamente $^{16}$. Grimm et al. en una serie de 125 pacientes tratados con braquiterapia exclusiva entre 1988 y 1990 reportan una supervivencia libre de recidiva bioquímica a diez años del $87 \%$. Hay que notar que en dicha serie la mayoría de los pacientes eran de bajo riesgo aunque un $23 \%$ de los mismos se presentaron con valores de PSA > $10 \mathrm{ng} / \mathrm{ml}^{17}$. Radge et al. publica una serie de 229 pacientes tratados entre 1987 y 1989. La estratificación de riesgo es como sigue: pacientes de bajo riesgo (Gleason score $<7$ y estadio clínico < T2b), que fueron tratados con braquiterapia exclusiva y pacientes de alto riesgo (Gleason score $>6$ o estadio clínico $>$ T2a) que fueron tratados con una combinación de radioterapia externa y braquiterapia. Los resultados de supervivencia libre de recidiva bioquímica actuariales a 12 años fueron de 66\% para el grupo de bajo riesgo y de 79\% para el grupo de alto riesgo ${ }^{2,18,19}$.

El seguimiento de los pacientes tratados con implantes permanentes representa uno de los puntos de controversia de la braquiterapia prostática. Dado que la próstata permanece "in situ" después de la braquiterapia, la interpretación de los niveles de PSA difiere de cuando se realiza una prostatectomía radical. Basándonos en series de pacientes tratados con radioterapia externa y con braquiterapia con seguimientos prolongados parece que aquellos que consiguen un nivel de PSA $<1,0 \mathrm{ng} / \mathrm{ml}$ y lo mantienen lograran tener supervivencia libre de enfermedad prolongada.

Aunque los resultados de la braquiterapia son comparables a los de prostatectomía radical y radioterapia externa, la aceptación de esta modalidad de tratamiento no es universal. Esto es debido a las continuas controversias en definir el fallo bioquímico. Mientras que la definición de la ASTRO de tres aumentos consecutivos de PSA está ampliamente extendida después de radioterapia externa, esto es cuestionado después de braquiterapia. Algunos investigadores han sugerido que sería más adecuado el conseguir un nadir de $0,5 \mathrm{ng} / \mathrm{ml}^{3,15,17,19}$.

Otro factor que confunde la determinación de fallo bioquímico es la aparición de "spikes" o picos de PSA después de braquiterapia. Se ha observado que un $35 \%$ de pacientes experimentan pequeños y temporales aumentos de PSA entre los 13 y 48 meses postimplante. El mecanismo de este fenómeno es desconocido pero se cree que puede ser debido a la respuesta tardía de los tejidos a la radiación. La magnitud de estas elevaciones está en torno a los $2 \mathrm{ng} / \mathrm{ml}$, aunque ocasionalmente pueden ser mayores.

La aparición de un pico de PSA no parece conferir un pronóstico adverso y podría no estar relacionado con ningún parámetro de la enfermedad o del tipo de tratamiento. Es importante reconocerlo para evitar terapias innecesarias ${ }^{20}$.

$\mathrm{El}$ papel del bloqueo hormonal en la braquiterapia prostática no está todavía establecido. Se sabe que el uso neoadyuvante de la deprivación hormonal puede disminuir el volumen prostático 
permitiendo un mejor acceso a la próstata sin interferencia del arco púbico. Sin embargo, su papel como tratamiento adyuvante en el control bioquímico de la enfermedad no está bien establecido.

En un reciente estudio retrospectivo de casoscontrol, Potters et al. no logra identificar ninguna ventaja con la adición de bloqueo hormonal neoadyuvante. Con un seguimiento medio de 46 meses, la probabilidad de control bioquímico de la enfermedad no presenta diferencias significativas entre el grupo de pacientes tratados con o sin hormonoterapia previa ${ }^{21}$. Merrick et al. aportan diferencias significativas en la probabilidad de supervivencia libre de recidiva bioquímica. Sin embargo estratificando por grupos de riesgo no encuentran diferencias significativas entre los tratados con o sin hormonoterapia ${ }^{22}$.

Sin embargo, Lee et al. reportan su experiencia en pacientes de riesgo intermedio o alto con la utilización de hormonoterapia adyuvante. La probabilidad de supervivencia libre de enfermedad a los cinco años era de 79\% y 54\% en el grupo de pacientes con y sin tratamiento hormonal $(\mathrm{p}<0,05)$ resultando en una probabilidad de supervivencia libre de recidiva bioquímica a cuatro años del 94\% para los pacientes de riesgo intermedio. En el estudio multivariado la hormonoterapia fue el único factor pronóstico ${ }^{23}$.

De nuevo, Merrick et al. analizan posteriormente la serie de pacientes de riesgo intermedio $y$ alto riesgo $(n=350)$ tratados mediante monoterapia con implante permanente $(n=57)$ o terapia combinada con radioterapia externa e implante permanente $(n=293)$. Del total de pacientes, 141 recibieron tratamiento hormonal. Para el grupo de riesgo intermedio no existieron diferencias significativas entre el grupo virgen de tratamiento hormonal y el grupo que recibió dicho tratamiento. En el grupo de alto riesgo la probabilidad de supervivencia libre de recidiva bioquímica a los 6 años fue del 79\% y 92\% para pacientes sin y con tratamiento hormonal respectivamente $(\mathrm{p}=0,046)^{24}$.

\section{CONCLUSIÓN}

Aunque el tratamiento de elección del cáncer de próstata organoconfinado sigue siendo la cirugía, otros métodos menos invasivos como la braquiterapia prostática tienen, sin duda, un lugar propio que la evolución posterior debe establecer. Es un tratamiento complejo que requiere la coordinación de un equipo multidisciplinar y una selección adecuada de los casos. Evita la prolongada hospitalización del paciente y permite una rápida recuperación así como de su actividad normal. Aunque nuestra serie es reciente y el seguimiento es escaso para poder establecer conclusiones de supervivencia y toxicidad, los datos apuntan hacia un buen control de la enfermedad superponible a los descritos en la literatura. No está exenta de efectos secundarios, aunque éstos son bien tolerados, de manejo asequible y pronta resolución.

\section{Agradecimientos}

"Este trabajo ha sido financiado con una beca de la Agencia D’Àvaluació de Tecnologia i Recerca Medica (AATRM) Proyecto 086/24/2000”.

\section{REFERENCIAS}

1. Blasko J, Radge H. Transperineal ultrasound guided implantation of the prostate: morbidity and complications. Scand J Urol Nephol Suppl 1991;137:113-118.

2. Radge H, Korb LJ, Elgamal AA, Grado GL, Nadir BS. Modern prostate brachytherapy. Prostate specific antigen results in 219 patients with up to 12 years of observed follow-up. Cancer 2000;89:135-141.

3. Radge $\mathrm{H}$, Korb L. Brachytherapy for clinically localized prostate cancer. Semin Surg Oncol 2000;18:45-51.

4. Oliva G. Braquiterapia en cáncer de próstata. Centro de publicaciones. Ministerio de Sanidad y Consumo. Madrid, noviembre 2000.

5. Parkin DM, Whelan S, Ferlay J, Raymond L, Young J, editores. Cancer Incidence in Five Continents. Volume VII. Lyon: International Agency for Research on Cancer 1997.

6. Berenguer A.: "Sgreening” del cáncer de próstata: un problema no resuelto. Actas Urol Esp 1996;20:768-771.

7. Partin AW, Kattan MW, Subong En et al. Combination of prostate-specific antigen, clinical stage, and Gleason score to predict pathological stage of localized prostate cancer. A multi-institutional update. JAMA 1997;277.

8. Nag S, Bice W, Dewyngaert K, Prestidge B, Stock R, Yu Y .The American Brachytherapy Society recommendations for Permanent prostate brachytherapy postimplant dosimetric analysis. Int $\mathrm{J}$ Radiation Oncology Biol Phys, 2000;46(1): 221-230.

9. Zelefsky MJ, Hollister T, Raben A, Matthews S, Wallner KE. Fiev year biochemical outcome and toxicity with transperineal CT-planned permanet $\mathrm{I}^{125}$ prostate implantation for patient with localized prostate cancer. Int $\mathrm{J}$ Radiation Oncology Biol Phys 2000;47(5):1261-1266.

10. Ash D, Flynn A, Battermann J, Reijke T, Lavagnini P, Blank L. ESTRO/EAU/EORTC recommendation on permanent seed implantation for localized prostate cancer. Int J Radiation Oncology Biol Phys 2000;57:315-321. 
11. Nag S, Beyer D; Friedland J, Grimm P, Nath R. American Brachytherapy society (ABS) recomendations for transperineal permanent brachytherapy of prostate cancer. Int $\mathrm{J}$ Radiation Oncology Biol Phys 1999; 44(4):789-799.

12. Yu Y, Anderson LL, Li Z, Mallenberg DE, Nath R, Schell MC, Waterman FM, Wu A, Blasko JC. Permanent prostate seed implant brachytherapy: Report of the American Association of Physicist in Medicine Task Group no 64 . Med Phys 1999; october;26(10).

13. Aguiló F, Suarez JF, Guedea F, Pera J, Cinos C, Oliva G, Serrallach N. Indicaciones y Tècnica de la Braquiterapia prostática. Actas Urol Esp 2002;26:24-26.

14. Nath R, Anderson LL, Luxton G, Weaver KA, Williamson JF, Meigooni AS. Dosimetry of interstitial brachytherapy sources: recomendations of the AAPM. Radiation Therapy Committe Task Group no 43 Med Phys 1995 february:22 (2).

15. Blasko JC, Mate T, Sylvester JE, Grimm PD, Cavanagh W. Brachytherapy for Carcinoma of the prostate: Technique, patient selection and clinical outcome. Seminars in Radiation Oncology January 2002;12(1):81-94.

16. Kollmeier MA, Stock Rg, Stone N.Biochemical outcomes after prostate brachytherapy with five minimal follow up: importance of patient selection and implant quality. Int $\mathrm{J}$ Radiation Oncology Biol Phys 2003;57(3):645-53,.

17. Grimm P, Blasko JC, Sylvester J, Meier R, Cavanagh W. 10-year biochemical (prostate-specific antigen) control of Prostate cancer with $\mathrm{I}^{125}$ brachytherapy. Int $\mathrm{J}$ Radiation Oncology Biol Phys 2001;51(1):31-40.

18. Radge H, Blasko JC, Grimm PD, Kenny GM, Sylvester JE, Hoak DC, Landin K, Cavanagh W. Intersticial iodine-125 radiation without adjuvant therapy in the treatment of clinically localized prostate carcinoma. Cancer August 1997;87(3):442-453.

19. Radge H, Elgamal A, Snow PB, Brandt J, Bartolucci A, Nadir BS, Korb LJ. Ten-year disease free survival after transperineal sonography-guided iodine-125 brachytherapy with or without 45 Gray external beam irradiation in the treatment ot patient with clinically localized, low to high Gleason grade prostate carcinoma. Cer September 1998;83(5):989-1001.

20. Stock R, Stone N. and Cesaretti J. Prostate-specific antigen bounce after prostate seed implantation for localized prostate cancer: descriptions and implications. Int $\mathrm{J} \mathrm{Ra-}$ diation Oncology Biol Phys 2003;56(2):448-453.
21. Potters L, Torre T, Ashley R, Leíble S. Examining the role of neoadyuvant androgen deprivation in patient undergoing prostate brachytherapy. Journal of Clinical Oncology March 2000;18(6): 1187-1192.

22. Merrick G, Butler W, Galbreath R, Lief J, PH.D. Five-year biochemical outcome following permanent Interstitial brachytherapy for clinical t1-t3 Prostate cancer. Int $\mathrm{J}$ Radiation Oncology Biol Phys 2001;51(1):41-48.

23. Lee LN, Stock RG, Stone NN. Role of hormonal therapy in the management of intermediate-to high risk prostate cancer treated with permanent radioactive seed implantation. Int J Radiation Oncology Biol Phys 2002;52(2):444-452.

24. Merrick GS, Butler WM, Galbreath RW, Lief JH, Adamovic E. Does Hormonal manipulation in conjuntion with permanet intersticial brachytherapy, with or without supplemental external beam irradiation, improve the biochemical outcome for men with intermediate or high risk prostate cancer? BJU Int Jan 2003;91(1):23-29.

25. Wallner K, Merrick G, True L, Sutlief S, Cavanagh W, Butler W. $\mathrm{I}^{125}$ versus Pd103 for low-risk prostate cancer: preliminary psa outcomes from a prospective randomized multicenter trial. Int. J Radiation Oncology Biol Phys 2003; 57(5): 1297-1303.

26. Yoshioka Y, Nose T, Yoshida K, Oh R, Yamada Y, Tanaka E, Yamazaki H, Inoue T, Inoue T. High-dose-rate brachytherapy as monotherapy for localized prostate cancer: a retrospective analysis with special focus on tolerance and chronic toxicity. Int J Radiation Oncology Biol Phys 2003;56(1):213-220.

27. Grills I, Martinez A, Hollander M, Huang R, Goldman K, Chen P, Gustafson G. High dose rate brachytherapy as prostate cancer monotherapy reduces toxicity compared to low dose rate palladium seeds. Journal of Urology 2004;171:1098-1104.

\author{
Dr. F. Aguiló Lúcia \\ Servicio de Urología \\ Hospital Universitari de Bellvitge \\ C/ Feixa Llarga s/n. \\ 08907 Hospitalet de Llobregat (Barcelona)
}

(Trabajo recibido el 11 mayo de 2004) 